Meta

Journal des tradlucteurs

Translators' Journal

\title{
Fondements linguistiques de la terminologie
}

\section{Christine Portelance}

Volume 36, numéro 1, mars 1991

La terminologie dans le monde : orientations et recherches

URI : https://id.erudit.org/iderudit/003859ar

Aller au sommaire du numéro

Éditeur(s)

Les Presses de l'Université de Montréal

\section{ISSN}

0026-0452 (imprimé)

Découvrir la revue

Citer cet article

Portelance, C. (1991). Fondements linguistiques de la terminologie. Meta, 36(1),

64-70. d'utilisation que vous pouvez consulter en ligne.

https://apropos.erudit.org/fr/usagers/politique-dutilisation/ 


\title{
FONDEMENTS LINGUISTIQUES DE LA TERMINOLOGIE
}

\author{
Christine Portelance \\ Université McMaster, Hamilton, Canada
}

Le hasard est un ensemble de facteurs qui interviennent ou paraissent intervenir dans un processus, mais dont nous ne savons pas, définitivement peut-être, préciser l'action.

Albert Jacquard, Éloge de la différence.

\section{INTRODUCTION}

La terminologie en tant que discipline apparaît comme un phénomène relativement récent. En effet, le mot terminologie désigne l'ensemble des mots techniques appartenant à une science, un art, à un chercheur ou à un groupe de chercheurs ${ }^{1}$ bien avant de désigner l'étude systématique de «termes» ou mots et syntagmes spéciaux servant à dénommer classes d'objets et concepts ${ }^{3}$. Pourtant, dès 1837, dans un ouvrage intitulé Histoire des sciences inductives, William Whewell ${ }^{2}$ donne une définition de ce mot qui laisse présager l'émergence de cette nouvelle discipline: «systèmes de termes employés dans la description des objets de l'histoire naturelle». En effet, les notions «modernes» de système, objet et science reliées à celle de terme apparaissent comme les prémisses d'une théorie. Cependant, au XIX ${ }^{e}$ siècle, il ne semble pas exister d'appellation pour désigner l'étude systématique de termes et la théorie qui en découle. En français, il faut attendre les années dix-neuf cent soixante avant de voir le mot terminologie employé dans ce sens $^{3}$ alors que cet emploi existait en russe et en allemand depuis déjà quelques décennies. Encore de nos jours, la terminologie comme discipline n'est pas très bien reconnue en dehors des cercles de spécialistes. Il faut admettre que dans ce domaine les travaux théoriques ainsi que les publications ne pullulent pas; aussi, entreprendre une recherche théorique représente encore aujourd'hui un travail de pionnier.

Dans la pratique (rédaction, traduction et terminographie), les impératifs de la profession permettent rarement de poser la question du statut linguistique du terme. Aux prises avec un problème de vocabulaire et devant la mutité trop fréquente des ouvrages terminographiques, il n'est souvent d'autre ressource que de se tourner vers son propre jugement linguistique en pestant contre l'insuffisance des moyens disponibles.

La traduction a longtemps été perçue comme un art que l'on acquiert par la pratique. Or, depuis les années soixante, à l'époque d'une véritable explosion technologique, la traduction a pris le chemin de l'école. En effet, un peu partout dans le monde, des écoles de traduction dispensent maintenant un enseignement universitaire, admettant du coup la possibilité d'intégrer la recherche à la pratique. D'artisan qu'il était, l'apprenti traducteur doit maintenant se faire technicien de la langue pour éviter d'être pris de vertige dans les virages technologiques, et pour être en mesure de soutenir la concurrence sur le marché des industries de la langue. Dans ce monde transformé désormais en «village global», l'accroissement de la productivité est essentiel si l'on veut éviter l'usage de l'anglais comme lingua franca dans le domaine des communications spécialisées. 
Dans le contexte nord-américain, la terminologie s'est développée afin de répondre aux problèmes de vocabulaire propres à la traduction spécialisée et à la francisation des rnilieux de travail bilingues, créant dans son sillage la terminographie et la terminotique.

\section{QU'EST-CE QU'UN TERME?}

On peut envisager de définir le terme en le considérant sous différents angles; la terminologie dispose actuellement d'une définition pragmatique:

unité signifiante constituée d' un mot (terme simple) ou de plusieurs mots (terme complexe) et qui désigne une notion de façon univoque à l' intérieur d' un domaine 4 .

La définition des concepts clés (unité signifiante, désignation, univocité et domaine) participe à la fois de la linguistique, de la philosophie du langage et de l'épistémologie. Personne ne niera, en effet, que la notion domaine (sphère spécialisée de 1'expérience humaine ${ }^{5}$ ) est du ressort de l'extralinguistique. Vu cet état de faits, on est en droit de s'interroger sur la légitimité du terme comme objet d'étude linguistique.

D'une part, peu de linguistes se sont intéressés à la terminologie, l'étude du lexique n'ayant jamais occupé une place prépondérante en linguistique théorique et ce, depuis le début du structuralisme. D'autre part, certains comme Coseriu (1967), Rey (1979) et Le Guern (1989) estiment que les considérations extralinguistiques dans la formation des termes sont trop importantes pour faire de la terminologie une branche spécialisée de la lexicologie.

Or, pour opposer lexicologie et terminologie, on avance généralement qu'un lexème est constitué d'un signifiant et d'un signifié alors qu'un terme entretient une relation privilégiée avec le référent. Ce serait donc le rapport à la chose qui distinguerait le terme du mot. Nous ne croyons pas que cet argument résiste à l'analyse.

Pour reprendre un débat aussi vieux que le Cratyle de Platon, il faut se garder de confondre les mots et les choses. La liste des termes d'un domaine atteste l'existence d'un discours relatif à ce domaine et c'est dans le discours qu'il faut chercher la référence et non pas dans le terme lui-même. En est-il vraiment autrement du lexique? Un lexème peut-il exister sans jamais être actualisé? Qu'est-ce que le concept de langue sans celui de la parole? La langue n'est pas la parole, mais historiquement le fait de parole précède toujours.

La notion d'atome a beaucoup évolué depuis Démocrite sans qu'on ait senti le besoin d'en changer l'appellation; évoquer cet exemple est maintenant devenu un lieu commun en terminologie. Les mathématiques ont servi de langage de démonstration aux sciences dites «dures»; pourtant le modèle actuel en physique des particules est incohérent du point de vue des mathématiques sans pour autant avoir été rejeté. Les limites de l'adéquation des mathématiques à fournir une modélisation cohérente des observables sont fixées par notre propre capacité à déterminer ces observables et ainsi construire notre monde scientifique. Depuis la «découverte» du principe d'incertitude d'Heisenberg, on peut même se demander avec une pointe d'humour si les mathématiques pures ne sont pas à la science ce que la poésie est à la langue de tous les jours. L'ancrage dans la réalitế de certaines notions de la physique actuelle n'est peut-être pas plus solide que celui des licornes et des sirènes de la mythologie.

Entre deux théories (discours?) successives ou concurrentes, les chercheurs utilisent souvent les mêmes termes, mais les rapports qu'entretiennent entre eux ces termes peuvent être différents. En demandant, par exemple, à deux éminents chercheurs, un chimiste et un physicien, si un atome d'hélium constitue une molécule, on obtint deux réponses catégoriques mais différentes. Pour le chimiste, oui sans hésitation, puisque cet atome se comporte comme une molécule du point de vue de la théorie des gaz. Pour le 
physicien, non indéniablement, puisque cet atome ne permet pas d'apercevoir un spectre moléculaire. Lorsque les partisans de Copernic niaient au Soleil le statut de planète, ils n'apprenaient pas ce qu'était une planète ou le soleil, ils redéfinissaient le mot planète. La loi d'Ohms ne pouvait être juste sans une redéfinition des termes courant et résistance ${ }^{6}$.

On peut avancer comme contre-argument que la langue de la science n'est pas la langue de la technique. En examinant l'interaction science et technique, on peut observer une imbrication certaine : dans certains cas, la technique précède la science (la machine à vapeur a précédé la thermodynamique; la sélection des races animales, la génétique). Par contre, les industries électriques se sont développées à partir de la connaissance des lois fondamentales de l'électricité. De plus, c'est la technique qui fournit à la science ses moyens d'investigation puisque les chercheurs doivent souvent concevoir leurs appareils d'investigation (ex.: les accélérateurs de particules).

Guilbert (1965) a montré que le vocabulaire d'une nouvelle spécialité se constitue surtout à partir de néologismes d'emploi; son étude montre que le vocabulaire de l'aviation s'est formé à partir d'un transfert de termes puisés dans les domaines de la physique, des mathématiques, de l'astronomie, de la mécanique, de l'entomologie, de l'ichtyologie, de la marine et du chemin de fer. Dans sa conclusion, Guilbert (1965: 332) affirme la prédominance des rapports linguistiques sur la structuration de l'expérience :

«Les formes lexicales qui assurent pourtant le relai (sic) direct entre l'expérience du monde et la langue sont régies par des rapports spécifiquement linguistiques qui ne s'identifient pas directement à la structuration de l'expérience.»

En fait, la dichotomie langue générale/langues de spécialité se fait à partir de l'opposition expérience partagée par l'ensemble des locuteurs/expérience partagée par des sous-ensembles de locuteurs. Toutefois, dans un cas comme dans l'autre, l'opération de définition est la même, elle consiste à établir les conditions d'utilisation des unités (mots ou termes) dans le(s) discours.

\section{TERMINOLOGIE ET SYNTAGMATIQUE}

Comme on le sait, la majorité des termes d'une nomenclature sont constitués de syntagmes (Baudot 1988; Boulanger 1988; Portelance 1987, 1989; entre autres). Nous avons décidé de procéder à l'étude du syntagme terminologique d'un point de vue linguistique. Par conséquent, nous considérons la dénomination (naming en anglais) comme le mécanisme linguistique du processus de la désignation. Il s'agit pour nous de tenter de dégager les structures linguistiques de la dénomination.

$\mathrm{Au}$ moment d'entreprendre cette étude de syntagmatique, il n'existait aucune recherche théorique récente sur le syntagme terminologique français (cf. Boulanger 1988). En effet, depuis vingt ans, les questions théoriques en terminologie n'ont guère progressé en l'absence d'un appareil d'investigation adapté à l'objet d'étude. En acceptant le caractère exploratoire de notre entreprise, une seule voie s'offrait à nous: le travail par induction. Comme point de départ, nous avions un ensemble de questions formulées à partir d'intuitions développées au contact de données quantitatives sur la saturation du vocabulaire observée dans des lexiques spécialisés de tailles et de factures différentes (Baudot 1988). Or, il était impossible de remonter des faits aux règles sans appareil d'investigation; aussi, tout notre travail de réflexion a été conditionné par les rapports intimes qu'entretiennent en recherche théorie et méthodologie, les deux faces de notre problématique. 


\section{MÉTHODOLOGIE}

Nous avons choisi de nous attaquer à un corpus de plus de 8000 termes complexes, puisés dans le domaine de l'électrotechnique. Ces termes sont normalisés, ce qui nous évitait de mettre en doute la légitimité des formes étudiées. Nous disposions également d'un corpus de textes (5000 mots) dans le même domaine.

En choisissant une démarche inductive, nous n'avions d'autre choix que de mettre à profit un vieil adage cartésien: séparer le tout en autant de parties qu'il faut pour le comprendre. Nous avons divisé notre sujet en différents objets d'étude: description lexico-syntaxique des termes en considérant les plans syntagmatique et paradigmatique, description lexico-sémantique en tenant compte du point de vue épistémologique et des deux plans cités précédemment, et enfin, confrontation des résultats de nos observations avec les manifestations terminologiques repérées en situation discursive.

Pour la description lexico-syntaxique, devant le nombre considérable de termes, nous avons procédé à un examen par tranche, en utilisant comme caractéristique de classement le nombre de substantifs présents dans chaque terme. Chaque classe de termes est examinée à partir d'une matrice théorique de description établie à l'aide de la liste de toutes les configurations du corpus (293 en tout).

Chaque matrice théorique de description permet de reproduire toutes les configurations d'une classe de termes. Chaque configuration constitue une matrice terminogénique. Les matrices terminogéniques d'une classe de termes forment la liste des règles de formation des termes du corpus appartenant à cette classe; elles sont classées par ordre de fréquence.

Chaque matrice théorique de description est constituée d'un certain nombre d'éléments dont au moins un est obligatoire. Ces éléments correspondent aux catégories lexicales attribuées à chaque constituant d'un terme. La répétition d'un élément dans une matrice de description correspond soit à la répétition dans une matrice terminogénique d'un constituant de même catégorie, soit aux différentes places qu'il peut occuper dans un syntagme, soit à la combinaison des deux phénomènes.

Chaque classe de termes possède un certain nombre d'éléments obligatoires: classe des mononominaux, un $\mathrm{N}$; classe des binominaux, deux $\mathrm{N}$; etc. Tous les autres éléments sont facultatifs, non parce que toutes les combinaisons sont possibles - il ne faut pas confondre ce type de matrice avec les matrices à deux dimensions utilisées en phonologie - mais parce qu'ils sont susceptibles d'apparaître à différents endroits dans une chaîne d'éléments. Une matrice théorique de description est formée, en fait, d'une suite de traces laissées par la somme des chaînes d'éléments constituant les matrices terminogéniques d'une classe de termes (Portelance 1986).

Étant donné la nature des matrices théoriques de description, les commentaires sur les contraintes de formation ne sont pas faits à partir de la liste exhaustive des combinaisons non réalisées, puisque nous prenons pour acquis qu'une liste du type :

$[\mathrm{N}+$ Prép + Prép];

$[\mathrm{N}+$ Prép + Prép + Prép];

$[\mathrm{N}+\mathrm{Adv}+$ Prép $]$

$[\mathrm{N}+$ Prép + Adv $]$

etc.

n'a de valeur descriptive que pour un automate puisqu'elle exprime les contraintes connues de la syntaxe générale. Par conséquent, les commentaires sur les contraintes de formation se consacrent aux formes possibles non réalisées et aux conditions de dépendance des éléments entre eux. 
Pour l'étude des manifestations terminologiques dans le discours, nous avons sélectionné des paramètres discursifs à partir du schéma de la communication suivant:

émetteur $\longrightarrow$ discours $\longrightarrow$ récepteur

Nous avons établi trois types d'émetteurs: l'auteur, le rédacteur et le traducteur, et trois types de récepteurs: le client, le terminographe et le linguiste.

L'ensemble de paramètres en rapport avec le discours sert à l'examen des règles d'organisation du discours. Chaque texte de notre corpus (1 000 mots) traite d'un produit, d'une notion du point de vue terminologique, cette notion constitue le thème autour duquel gravitent des rhèmes. Nous portons notre attention sur trois types de rhèmes: l'absence ou la présence d'une définition du thème, les notions périphériques, l'absence ou la présence de définitions des notions périphériques. L'examen des formes de reprise du thème permet de traiter de l'opposition syntagme terminologique/syntagme libre, de la réduction de syntagmes terminologiques et de l'existence de stratégies terminologiques de discours.

\section{CONCLUSION}

Le syntagme terminologique est constitué d'une tête et d'une expansion formée d'une ou de plusieurs déterminations. D'un point de vue fonctionnel, tout élément servant de détermination à la tête du syntagme est un adjectif, lequel a un sens absolu. L'opposition syntagme terminologique et syntagme libre peut se faire à partir de l'identification de la relation existant entre la tête et son expansion adjectivale. Lorsque l'adjectif entraîne une modification du référent, nous sommes en présence d'un syntagme terminologique, lorsqu'il détermine une modification de la référence, il s'agit d'un syntagme libre (cf. Bolinger 1967). Du point de vue sémantique, la principale différence entre les réalisations adjectivales consiste en l'existence ou l'absence d'un référent potentiel. Dans un terme de forme $N+A d j$, la détermination Adj n'a pas de référent potentiel, elle n'a qu'une signification alors qu'une détermination Prép $+N$ possède et une signification et un référent potentiel. Lorsque les déterminations sont constituées de termes de la nomenclature, on peut considérer ces déterminations comme étant actualisées; dans le cas contraire et lorsque la détermination est constituée d'un substantif, avec ou sans préposition, le référent n'est que virtuel.

On ne peut procéder à l'analyse lexico-syntaxique des syntagmes terminologiques sans tenir compte du plan paradigmatique puisque ce plan est essentiel dans l'identification des niveaux d'enchâssement des déterminations. Cet état de fait est dû à l'existence des séries de termes et à la réutilisation de termes comme détermination de têtes de syntagmes. Les termes peuvent connaître deux sortes de rapports paradigmatiques avec les autres termes d'un ensemble de termes: des rapports paradigmatiques directs et des rapports paradigmatiques obliques (Portelance 1989).

Dans une série de termes à tête identique, les liens sémantiques des déterminations avec la tête ne peuvent être représentés par un système fermé de traits pertinents à cause de la dynamique de dénomination. Chaque détermination constitue une caractéristique différentielle, une information, qui, ajoutée à une tête, identifie une notion parmi un ensemble de notions. Lorsque la caractéristique n'est plus différentielle, la détermination devenant inutile tombe. Le mécanisme en jeu dans la réduction des syntagmes s'articule à partir d'un système vectoriel implicite/explicite. Chaque fois qu'un syntagme est ajouté à une série, le choix de la détermination s'opère en fonction des liens sémantiques déjà existants entre la tête et les déterminations de la série. Dans certains cas, l'inclusion d'un nouveau terme peut entrainer une modification de la valeur des déterminations présentes, 
puisque chaque fois qu'on ajoute une nouvelle détermination, c'est tout le système de discrimination qui s'en trouve transformé sans pour autant être bouleversé.

Dans une nomenclature, le phénomène des séries explique les statistiques sur la saturation du vocabulaire; en faisant une projection sur ces chiffres, on peut affirmer que si l'on devait nommer une nouvelle notion dans un domaine technoscientifique, il y a de fortes probabilités pour que le nouveau terme soit un syntagme et qu'au moins un des constituants soit déjà présent dans le vocabulaire de la nomenclature du domaine.

L'importance du phénomène des séries et des rapports paradigmatiques que cela implique nous amène à proposer que ces rapports soient partie intégrante de la définition d'un terme. Dans la définition de chaque terme d'une nomenclature, il faudrait pouvoir accéder à la liste des termes où le défini apparaît comme tête, et la liste des termes où il apparaît comme détermination. La connaissance des matrices terminogéniques permet de procéder automatiquement à la reconnaissance de ces deux types de rapports paradigmatiques. Ce nouveau type de définition permet de percer à jour le potentiel de dénomination d'une nomenclature et pourrait être fort utile au traducteur en panne d'équivalent.

La terminologie peut servir à déterminer certains des paramètres pertinents dans l'établissement d'une typologie des discours technoscientifiques. Nous émettons l'hypothèse qu'un texte scientifique ou technoscientifique est formé d'un réseau de relations entre différentes notions présentes dans un texte, avec ou sans dénomination, avec ou sans définition. Ces relations peuvent être plus ou moins implicites. Cette échelle de l'explicite à l'implicite, représentant la connivence entre émetteur et récepteur, peut être déterminée à partir du paramètre de densité terminologique d'un discours mis en rapport avec le nombre de notions définies et la présence de termes transparents et opaques.

$\mathrm{Au}$ bout de notre entreprise, une première étape est complétée, une étape taxinomique certes, mais essentielle. Pour procéder maintenant à la description lexicosyntaxique des termes d'une nomenclature, il faudrait retourner au point de départ, reprendre le traitement informatique des données pour obtenir à nouveau de l'information et tirer de nouvelles listes:

1. Une liste de chacun des termes accompagné des termes en rapport paradigmatique direct et en rapport paradigmatique oblique.

2. À partir de la première liste, pour chaque classe de termes, produire une liste identifiant les enchâssements dans toutes les matrices terminogéniques.

Exemples:

$[\mathrm{N}+$ Adj] + Prép $+\mathrm{N}]]$

$[\mathrm{N}+$ Prép $+\mathrm{N}]+$ Adj]

$[$ [ $\mathrm{N}+$ Prép $+[\mathrm{N}+$ Adj $]]$

3. Pour chaque classe de termes, compiler les matrices terminogéniques ainsi analysées et constituer des matrices théoriques cartographiques.

À partir de cette réorganisation des données, il devient possible d'étudier la limite de longueur d'un terme en se servant de l'hypothèse suivante: la longueur limite théorique d'un terme ne dépend pas du nombre d'éléments constituant les déterminations, ni du nombre de déterminations, mais des niveaux d'enchâssement des déterminations mis en rapport avec le nombre de déterminations.

Derrière cette démarche se profile une deuxième hypothèse qu'on peut rattacher à un modèle théorique: si l'on tient compte des niveaux d'enchâssement, la structure d'un syntagme terminologique possède des limites de génération similaires à celles d'une phrase. En effet, un terme constitué d'une tête et d'une expansion formée d'une série de 
déterminations en relation coordonnée peut, sur le plan théorique et sans tenir compte du discours, ne connaitre aucune limite de longueur, tout comme une phrase utilisant le procédé d'énumération, telle:

Hier soir, pour m'endormir, j' ai compté un mouton, deux moutons, trois moutons, etc.

C'est le critère d'acceptabilité qui, comme en syntaxe générale, détermine la longueur maximale. Après l'étude des niveaux d'enchâssement, si notre hypothèse se révélait fausse, elle pourrait au moins servir à poser le problème de la syntagmatique avec plus d'acuité; car si tel était le cas, la vérification d'une telle hypothèse permettrait indéniablement, si ce n'est à cause des ressemblances à tout le moins à cause des dissemblances, de rattacher cette syntagmatique à la syntaxe générale.

Même si nous n'avons qu'une réponse partielle à la question théorique de la syntagmatique, nous avons la conviction que l'appareil d'investigation que nous avons constitué a fait ses preuves et mérite d'être développé. Dans le cadre de notre travail, l'interaction entre méthodologie et théorie, leur interpénétrabilité montrent bien qu'on ne peut développer de modèle théorique sans méthodologie, mais on ne peut toutefois établir de méthodologie sans concepts de base. Kant avait bien raison, il semble qu'on ne peut se passer de catégories a priori.

\section{NOTES}

1. Définitions du Petit Robert.

2. Épistémologue et moraliste anglais (1794-1866) cité par Rey (1979: 7)

3. Dans le Petit Robert depuis 1978.

4. Vocabulaire systématique de la terminologie, OLF, 1985.

5. Définition tirée du vocabulaire mentionné précédemment.

6. Exemples empruntés à Kuhn (1970).

\section{BIBLIOGRAPHIE}

BAUDOT, J. (1988): «Une minibanque de terminologie multilingue», Terminogramme, 46. BOLINGER, D. (1967): «Adjectives in English: Attribution and Predication», Lingua, 18, pp. 1-34.

BOULANGER, J.-C. (1988) : «Le syntagme terminologique : un projet de recherche», Terminogramme, 46.

COSERIU, E. (1967): «Structures lexicales et enseignement du vocabulaire», Les théories linguistiques et leur application, AIDELA.

GOFFIN, R. (1973) : «Structures lexicales et terminologies multilingues», Meta, 18.

GUILBERT, L. (1965) : La formation du vocabulaire de l'aviation, Larousse.

HAIMAN, J. (1980): «Dictionaries and Encyclopedias», Lingua, 50.

KANT, E. (1976): Critique de la raison pure, Flammarion.

KUHN, T.S. (1970): La structure des révolutions scientifiques, Champs, Flammarion

LE GUERN, M. (1989) : «Sur les relations entre terminologie et lexique», Meta, 34-3.

LICHNEROWICZ, A. (1987): «Universalité des mathématiques et compréhension du réel», Les scientifiques parlent..., sous la direction de Albert Jacquard, Hachette.

POLANYI, M. (1958): Personal Knowledge. Towards a Post-critical Philosophy, The University of Chigago Press.

POLANYI, M. (1946): Introduction (1964) Science Faith and Society, The University of Chigago Press.

PORTELANCE, C. (1989): «Syntagme et Paradigme», Meta, 34-3.

PORTELANCE, C. (1987): «Fertilisation terminologique ou insémination terminologique artificielle», Meta, 32-3.

PORTELANCE, C. (1986) : «À propos du rôle des matrices terminogéniques...», Terminogramme, 39-40.

REY, A. (1979) : La terminologie : noms et notions, Coll. «Que-sais-je?», $\mathrm{n}^{\circ} 1780$, PUF.

SAUSSURE de, F. (1972): Cours de linguistique générale, Payot, nouvelle édition.

WIERZBICKA, A. (1985): Lexicography and Conceptual Analysis, Karoma Publishers. 